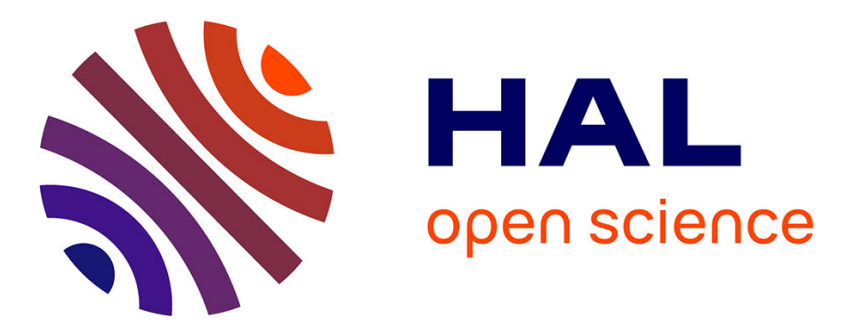

\title{
Acoustic characterization of porous materials with a rigid structure in the low frequency regime
}

Mustapha Sadouki, Mohamed Fellah, Zine El Abiddine Fellah, Erick Ogam, Claude Depollier

\section{- To cite this version:}

Mustapha Sadouki, Mohamed Fellah, Zine El Abiddine Fellah, Erick Ogam, Claude Depollier. Acoustic characterization of porous materials with a rigid structure in the low frequency regime. Fifth Biot Conference on Poromechanics, Jul 2013, Vienne, Austria. pp.193-199. hal-00868132

\section{HAL Id: hal-00868132 https://hal.science/hal-00868132}

Submitted on 2 Oct 2013

HAL is a multi-disciplinary open access archive for the deposit and dissemination of scientific research documents, whether they are published or not. The documents may come from teaching and research institutions in France or abroad, or from public or private research centers.
L'archive ouverte pluridisciplinaire HAL, est destinée au dépôt et à la diffusion de documents scientifiques de niveau recherche, publiés ou non, émanant des établissements d'enseignement et de recherche français ou étrangers, des laboratoires publics ou privés. 


\title{
Acoustic characterization of porous materials with a rigid structure in the low
} frequency regime.

M. Sadouki ${ }^{1}$, M. Fellah ${ }^{2}$, Z.E.A. Fellah ${ }^{3}$, E. Ogam ${ }^{3}$ and C. Depollier ${ }^{4}$

${ }^{1}$ Faculté des Sciences et Technique, Université de Khemis Miliana, Route de Thénia, Khemis Miliana BP 44225, Algérie, email: sad.mus17@ yahoo.fr

${ }^{2}$ Laboratoire de Physique Théorique, Faculté de Physique, USTHB, BP 32 El Alia, Bab Ezzouar 16111, Algérie, email: mfellah@usthb.dz

${ }^{3}$ LMA, CNRS, UPR 7051, Aix-Marseille Univ, Centrale Marseille, F-13402 Marseille Cedex 20, France, email: Fellah@1ma.cnrs-mrs.fr

${ }^{4}$ LUNAM Universite du Maine. UMR CNRS 6613 LAUM UFR STS Avenue O. Messiaen 72085 Le Mans CEDEX 09 France, email: Claude.Depollier@univ-lemans.fr

\begin{abstract}
An acoustic method is proposed for measuring the low frequencies parameters of porous materials having a rigid frame. The Biot equivalent fluid model is considered, in which the viscous losses are described by the the static viscous permeability and an additional viscous parameter. The inertial interactions between fluid and structure are taken into account by the inertial factor. The thermal couplings are modeled using the thermal permeability and the thermal tortuosity. The proposed method is based on a solution of the direct and inverse problems for the propagation of transient audible frequency waves in a homogeneous isotropic slab of porous material having a rigid frame. The physical parameters are determined from the solution of the inverse problem using experimental reflected and transmitted waves by porous samples, in a wave guide (pipe). The minimization of the objective function, defined as the difference between experiment and theory, is done in the time domain. Tests are performed using industrial plastic foams. Experimental and theoretical data are in good agreement. Furthermore, the prospects are discussed.
\end{abstract}

\section{INTRODUCTION}

The acoustic characterization of porous materials saturated by air (Allard and Atalla, 2009) such as plastic foams, fibrous, or granular materials is of great interest for a wide range of industrial applications. These materials are frequently used in the automotive and aeronautics industries and in the building trade. Acoustic damping in air-saturated porous materials is described by the inertial, viscous, and thermal interactions between the fluid and the structure Depending on the temporal component of the acoustic excitation (pulse duration), the relaxation times describing the fluid structure interactions are different. In the high frequency domain (Johnson et al, 1987), the 
inertial, viscous, and thermal interactions are modeled by causal integro-differential operators acting as fractional derivatives (Fellah and Depollier 2000) in the time domain. These interactions are taken into account, by the high approximation of the tortuosity for the inertial effects, and by the viscous characteristic length and thermal characteristic length for the viscous and thermal losses. In the low frequency domain, the inertial, viscous, and thermal interactions are described, respectively, by the inertial factor and the thermal tortuosity, the viscous and thermal permeability (Lafarge et al 1996, 1993). In addition to these parameters, the porosity is a key parameter playing an important role for all relaxation times. The determination of these parameters is crucial for the prediction of the sound damping in these materials.

\section{EQUIVALENT FLUID MODEL}

In the acoustics of porous materials, one distinguishes two situations according to whether the frame is moving or not. In the first case, the dynamics of the waves due to the coupling between the solid skeleton and the fluid is well described by the Biot theory (Biot 1956). In air-saturated porous media, the vibrations of the structure can be neglected when the excitation is not very important and the waves can be considered to propagate only in fluid. This case is described by the model of equivalent fluid which is a particular case of the Biot model, in which fluid-structure interactions are taken into account in two frequency response factors: dynamic tortuosity of the medium $\alpha(\omega)$ given by Johnson (Johnson et al, 1987) and revisited by Pride (Pride et al, 1993) and Lafarge (1993), the dynamic compressibility of the air in the porous material $\beta(\omega)$ given by Allard etal (1992) and Lafarge (1993). In the frequency domain, these factors multiply the fluid density and compressibility respectively and show the deviation from fluid behavior in free space as frequency changes. In the low frequency range, $\alpha(\omega)$ and $\beta(\omega)$ may be expanded in successive powers $(j \omega)^{n}$. Exact explicit expressions may be obtained for the coefficients, in terms of averages (of increasing order) of the velocity and temperature fields. At low frequencies, $\alpha(\omega)$ and $\beta(\omega)$ are given by:

$$
\begin{aligned}
& \alpha(\omega)=-\frac{\eta \phi}{j \omega \rho k_{0}}+\alpha_{0}+\frac{2 \alpha_{\infty}{ }^{4} k_{0}{ }^{3} \rho}{\eta \Lambda^{4} \phi^{3} p^{3}} j \omega+\ldots \\
& \beta(\omega)=\gamma+\frac{(\gamma-1) k_{0}^{\prime} P_{r} \rho}{\eta \phi} j \omega-\frac{\alpha_{0}^{\prime}(\gamma-1) k_{0}^{\prime 2} P_{r}^{2} \rho^{2}}{\eta^{2} \phi^{2}} \omega^{2}+\ldots
\end{aligned}
$$

In these equations, $j^{2}=-1, \phi$ is the porosity, $k_{0}$ the static permeability, $\alpha_{\infty}$ the tortuosity, $\Lambda$ the viscous characteristic length , $p$ is a geometrical parameter introduced by Pride etal (1993) and revisited by Lafarge (1993), $\gamma$ is the adiabatic constant, $\operatorname{Pr}$ the Prandtl number. $k_{0}^{\prime}$ is the thermal permeability equal to the inverse trapping constant of the solid frame (Lafarge et al 1996). $\alpha_{0}$ represents the low-frequency approximation of the tortuosity given by by Norris (1986) from homogenization theory. It should be noted that the third term written in Eq. 1: $\frac{2 \alpha_{\infty}^{4} k_{0}^{3} \rho}{\eta \Lambda^{4} \phi^{3} p^{3}} j \omega$ does not result from the exact expansion of $\alpha(\omega)$ and $\beta(\omega)$, this would involve a new geometrical parameter which 
has not been calculated and measured. This term results from using Pride et al' (1993) approximate model.

Rewrite the equation (1) so that the parameter $\alpha_{1}$ will be introduced in the last term;

$$
\alpha(\omega)=\frac{\eta \phi}{-j \omega \rho k_{0}}+\alpha_{0}+\frac{k_{0} \rho \alpha_{1}}{\phi \eta} j \omega .
$$

$\alpha_{1}$ is dimensionless parameter and describes the viscous exchange between fluid and structure at low frequencies. We will try to measure experimentally this parameter using transmitted waves by a porous material.

\section{DIRECT PROBLEM}

The direct scattering problem is that of determining the scattered field (transmitted and reflected) as well as the internal field (incident), that arises when a known incident field impinges on the porous material with known physical properties. To compute the solution of the direct problem one need to know the reflection and transmission coefficients of the porous medium. Consider a homogeneous porous material that occupies the region $0 \leq x \leq L$.

The expression of the transmission coefficient for a wave propagating inside a porous material is given by

$$
T(\omega)=\frac{2 D}{2 D \cosh (j q L)+\left(1+D^{2}\right) \sinh (j q L)},
$$

with

$$
q=\omega \sqrt{\frac{\rho_{f}}{K_{a}} \alpha(\omega) \beta(\omega)}, \quad D=\phi \sqrt{\frac{\beta(\omega)}{\alpha(\omega)}} .
$$

The incident $p^{i}(t)$ and transmitted $p^{t}(t)$ fields are related in time domain by the transmission scattering operator:

$$
p^{t}(x, t)=\int_{0}^{t} \tilde{T}(\tau) p^{i}\left(t-\tau-\frac{(x-L)}{c_{0}}\right) d \tau .
$$

$\tilde{T}(t)$ is the temporal operator of transmission (inverse Fourier transform of $T(\omega)$ ). Using the theoretical expression of the transmission coefficient (4) and the experimental transmitted data, we will try to solve the inverse problem for the acoustic characterization of the porous material.

\section{INVERSE PROBLEM}

The inverse problem is to find the following parameters: viscous permeability $k_{0}$, thermal permeability $k_{0}^{\prime}$, inertial factor $\alpha_{0}$, thermal tortuosity $\alpha_{0}^{\prime}$ and the 


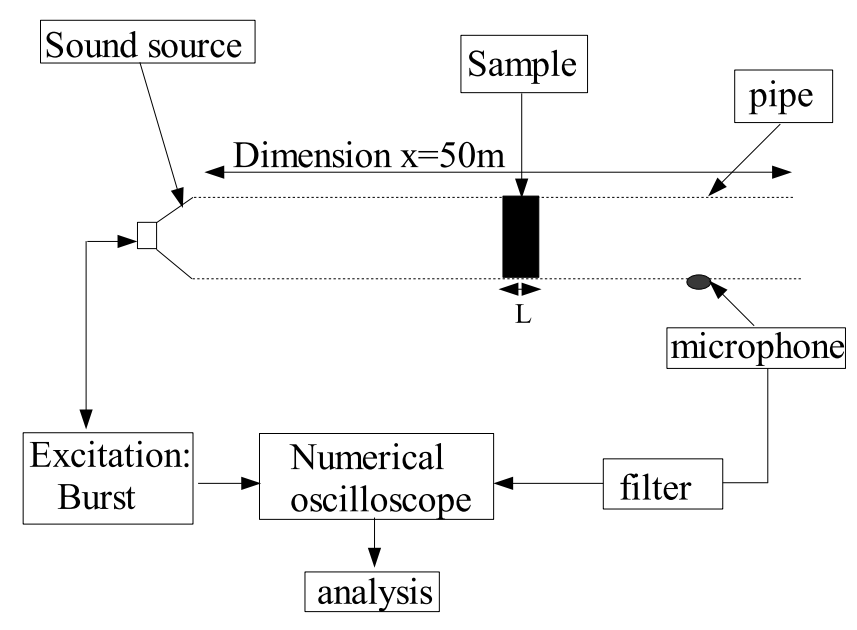

Figure 1. Experimental setup of acoustic measurements.

new viscous parameter $\alpha_{1}$ which minimize numerically the discrepancy function $U\left(k_{0}, k_{0}^{\prime}, \alpha_{0}, \alpha_{0}^{\prime}, \alpha_{1}\right)=\sum_{i=1}^{i=N}\left(p_{\text {exp }}^{t}\left(x, t_{i}\right)-p^{t}\left(x, t_{i}\right)\right)^{2}$, wherein $p_{\text {exp }}^{t}\left(x, t_{i}\right)_{i=1,2, \ldots n}$ is the discrete set of values of the experimental transmitted signal and $p^{t}\left(x, t_{i}\right)_{i=1,2, \ldots n}$ the discrete set of values of the simulated transmitted signal predicted from Eq. (6). The inverse problem is solved numerically by the least-square method. For its iterative solution, we used the simplex search method (Nedler Mead et al (1998)) which does not require numerical or analytic gradients.

Experiments are performed in a guide (pipe), having a diameter of $5 \mathrm{~cm}$. experimental set up is given in Fig. 1. A sound source Driver unit "Brand" constituted by loudspeaker Realistic $40-9000$ is used. Bursts are provided by synthesized function generator Standford Research Systems model DS345-30MHz. The signals are amplified and filtered using model SR 650-Dual channel filter, Standford Research Systems. The signals (incident and transmitted) are measured using the same microphone (Bruel\&Kjaer, 4190) in the same position in the tube. The incident signal is measured without porous sample, however, the transmitted signal is measured with the porous sample.

Consider a cylindrical sample of plastic foam $\mathrm{M}$ of diameter $5 \mathrm{~cm}$, porosity $\phi=0.95$ and thickness $5 \mathrm{~cm}$. The viscous permeability $k_{0}$ of the porous sample is measured using standard methods (Stinson et al 1988) obtaining the value of $k_{0}=(3 \pm 0.5) 10^{-9}$. Fig. 2 shows the experimental incident signal (solid line) generated by the loudspeaker and its spectrum. After solving the inverse problem numerically for the viscous $k_{0}$ and thermal permeability $k_{0}^{\prime}$, the inertial factor $\alpha_{0}$, the thermal tortuosity $\alpha_{0}^{\prime}$, and the new viscous parameter $\alpha_{1}$, we find the optimized values given in Table 1 . We present in Figs. 3 the variation of the minimization function $U$ with the inverted parameters. In Fig. 4, we show a comparison between an experimental transmitted 

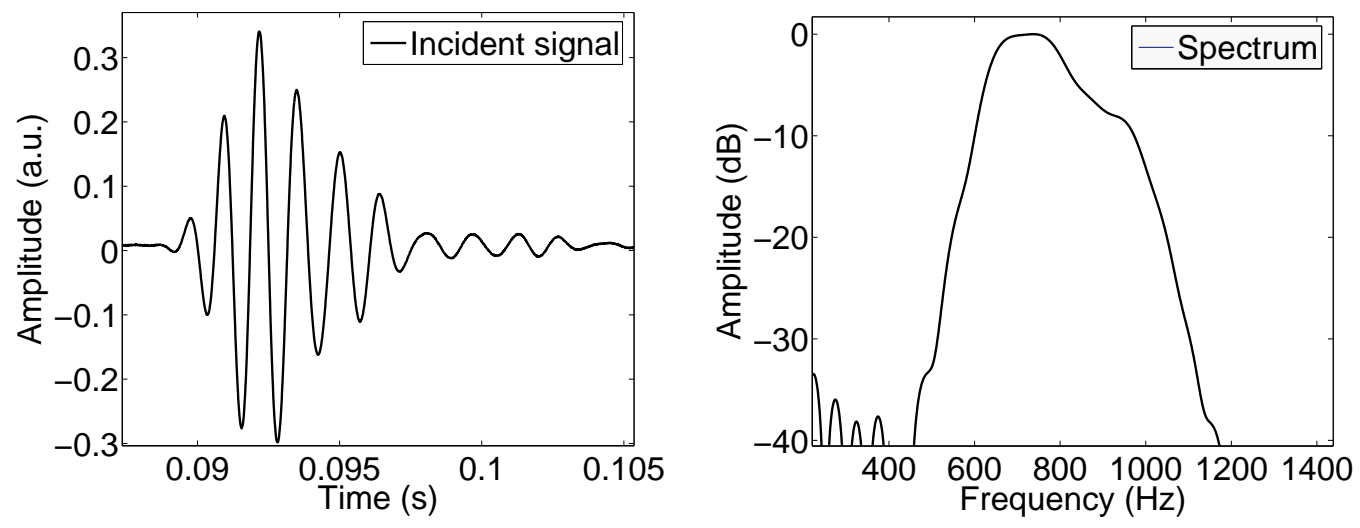

Figure 2. Incident experimental signal and its spectrum.

Table 1. Inverted parameters.

\begin{tabular}{llllll} 
Material & $k_{0}\left(10^{-9} \mathrm{~m}\right)$ & $\alpha_{0}$ & $k_{0}^{\prime}\left(10^{-9} \mathrm{~m}\right)$ & $\alpha_{0}^{\prime}$ & $\alpha_{1}$ \\
\hline Sample & 2.89 & 3.36 & 8.67 & 1.03 & 0.25 \\
\hline
\end{tabular}

signal and simulated transmitted signal for the optimized values of the physical parameters. The difference between the two curves is small with a slight temporal delay between the theoretical and experimental curves, probably due to the uncertainties of experimental measurements. This results leads us to conclude that the optimized values of physical parameters are correct. This study has been extended to other frequency bandwidths (1-3)kHz and has also given good results. The Biot's vibrations which induced structural disturbance resulting from elasticity, prevent applying the method for the resistive porous materials having a low viscous permeability value.

\section{CONCLUSION}

In this paper, a complete characterization of porous materials with rigid structure has been given in the low frequency range. A new parameter describing the viscous exchange between fluid and structure was introduced. The inverse problem has allowed to the experimental estimation of the physical parameters describing the propagation in this domain of frequency (viscous and thermal permeability, inertial parameter, thermal tortuosity and the new viscous parameter). The reconstructed value of viscous permeability is in agreement with this obtained using classical methods. The proposed experimental method has the advantage of being simple, rapid, and efficient for estimating those parameters and further characterizing porous materials. 

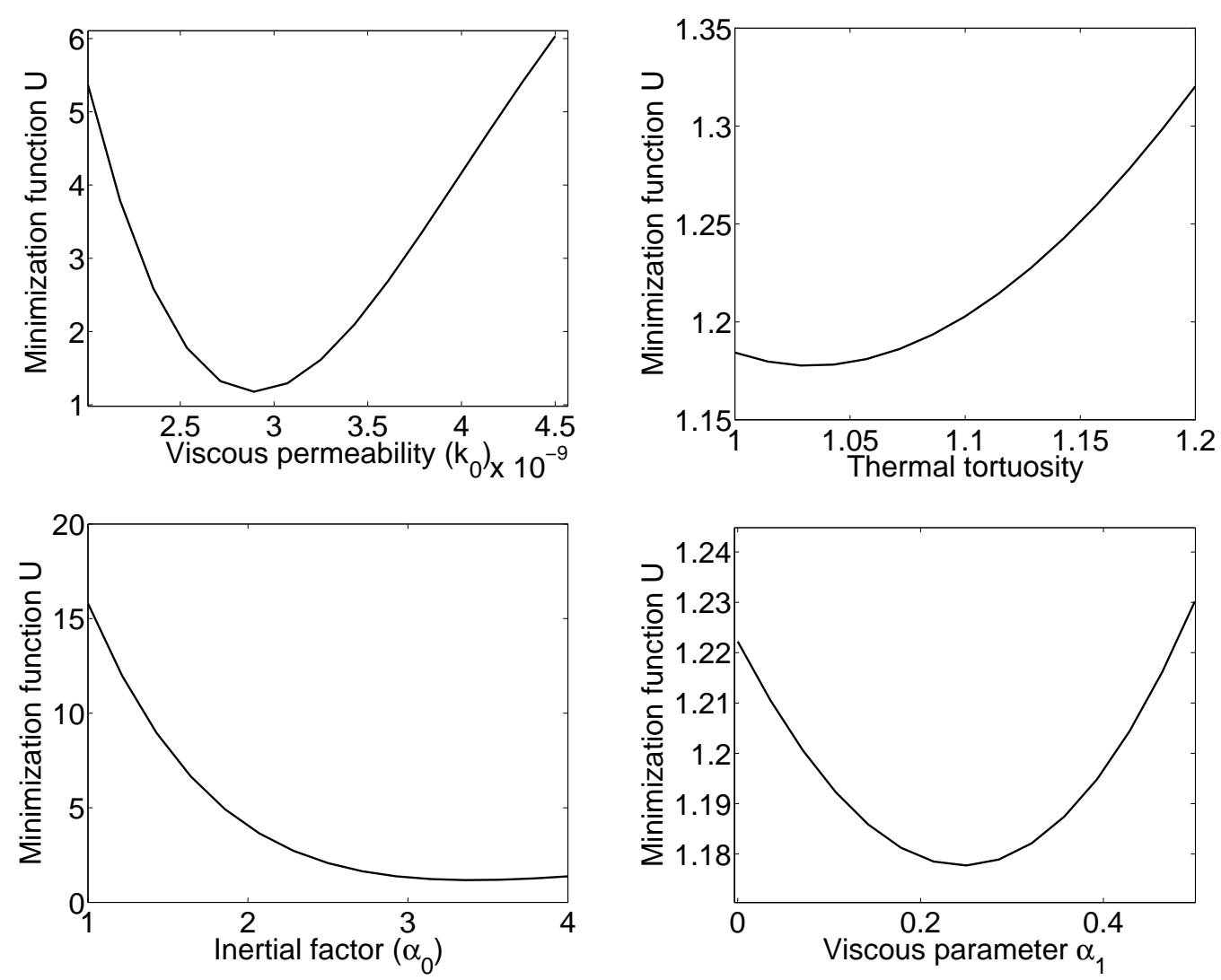

Figure 3. Minima of the inverse problem.

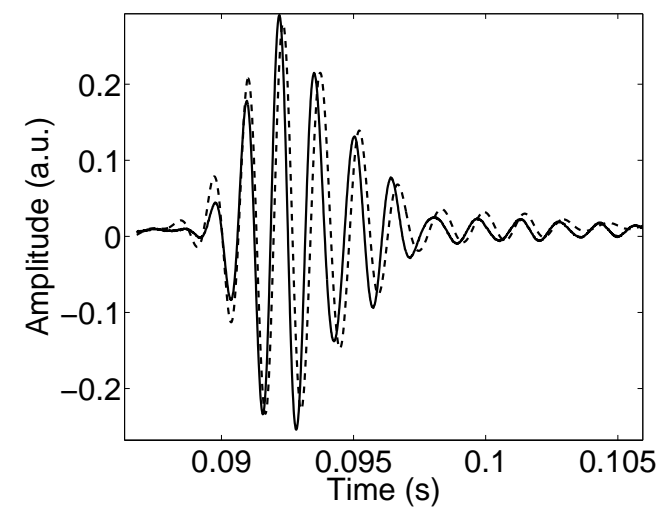

Figure 4. Comparison between the experimental transmitted signal (solid line) and the simulated transmitted signals (dashed line). 


\section{REFERENCES}

Allard J. F. and Atalla N., (2009) Chapter 5, Propagation of Sound in Porous Media: Modeling Sound Absorbing Materials J. Willey and Sons Ltd, Chichester, West Sussex, PO19 8SQ, United Kingdom.

Johnson D.L., Koplik J. and Dashen R. (1987), 'Theory of dynamic permeability and tortuosity in fluid-saturated porous media", J. Fluid.Mech. 176, 379-402.

Fellah Z.E.A and Depollier C. (2000), "Transient acoustic wave propagation in rigid porous media: A time-domain approach", J. Acoust. Soc. Am. 107, 683-688.

Lafarge D., Lemarinier P., Allard J. F. , Tarnow V. (1997), "Dynamic compressibility of air in porous structures at audible frequencies" J. Acoust. Soc. Am. 102, 19952006.

Biot M. A. (1956), 'The theory of propagation of elastic waves in fluid-saturated porous solid. I. Low frequency range", J. Acoust. Soc. Am., 28, 168-178.

Pride S.R. Moran F.D. Gangi A.F. (1993), "Drag forces of porous-medium acoustics", Phys. Rev. B. 47, 4964-4978.

Lafarge D. (1993), "Sound propagation in porous materials having a rigid frame saturated by gas", Ph.D. thesis, Universit du Maine, Le Mans, France.

Allard J.F., Champoux Y., (1992) "New empirical equations for sound propagation in rigid frame fibrous materials", J. Acoust. Soc. Am. 91, 33463353.

Norris A.N. (1986), "On the viscodynamic operator in Biot's equations of poroelasticity", J. Wave Mat. Interact. 1 365-380.

Lagarias J.C. , Reeds J.A., Wright M.H. and Wright P.E. (1998), "Convergence properties of the Nedler-Mead Simplex Method in low dimensions, "SIAM Journal of Optimization 9, 112-147.

Stinson M.R. and Daigle G.A. (1988), "Electronic system for the measurement of ow resistance," J. Acoust. Soc. Am. 83, 24222428. 